\title{
The Regularization Aspect of Optimal-Robust Conditional Value-at-Risk Portfolios
}

\author{
Apostolos Fertis and Michel Baes and Hans-Jakob Lüthi
}

\begin{abstract}
In portfolio management, Robust Conditional Value - at - Risk (Robust CVaR) has been proposed to deal with structured uncertainty in the estimation of the assets probability distribution. Meanwhile, regularization in portfolio optimization has been investigated as a way to construct portfolios that show satisfactory out-ofsample performance under estimation error. In this paper, we prove that optimalRobust CVaR portfolios possess the regularization property. Based on expected utility theory concepts, we explicitly derive the regularization scheme that these portfolios follow and its connection with the scenario set properties.
\end{abstract}

\section{Introduction}

The portfolio management problem refers to the decision on the proportion of wealth invested in a set of available assets such that the uncertain return is optimized according to some metric taking into account both its magnitude and the risk in its potential values. Markowitz was the first one to formulate a quantitative approach to this problem [17]. The mean-variance approach that he followed set the path for the development of the fields of financial engineering and asset pricing, drastically affected by the milestones of the Capital Asset Pricing Model [16, 19, 24], the Arbitrage Pricing Theory [22], the Black-Scholes model [3] and Merton's theory of option pricing [18].

Much concern has been expressed about the effects of errors in the estimation of the parameters that characterize the probability distribution of the asset returns. Constraining portfolio norms has been proposed as a technique to tackle this uncertainty in the estimated parameters with the resulting regularized portfolios showing improved out-of-sample performance $[8,9,13]$. Bayesian techniques have been also

Apostolos Fertis

Institute for Operations Research (IFOR), Eidgenössische Technische Hochschule Zürich, Rämistrasse 101, HG G 21.3, 8092, Zürich, Switzerland, tel.: +41 44632 4031, e-mail: afertis@ifor.math.ethz.ch 
suggested $[2,12]$. Estimation error insensitive portfolios derived through Huber's robust statistics methods [15] have been investigated [10,12].

Meanwhile, risk management has been connected with robust optimization. Robust optimization deals with uncertainty in the data of an optimization problem. It seeks the solution that optimizes the worst-case value of the objective function when the data vary in some uncertainty set. Such a solution can be efficiently computed for many cases of uncertainty sets in linear or general convex optimization problems $[4,5,7]$. Given that in portfolio management the parameters of the assets probability distribution are estimated with limited accuracy, their true value can be assumed to reside in an uncertainty set surrounding the estimated value. Based on problems that optimize a trade-off between the mean and the variance of the portfolio, corresponding robust optimization problems that optimize the worst-case trade-off when the probability distribution parameters vary in uncertainty sets have been proposed and solved [14]. The robust problems have been solved by transforming them to equivalent problems that impose regularization constraints on the portfolio weights [14].

Furthermore, risk measures have been considered to be mappings of random financial positions to real numbers imposing a preferential order among financial positions that encapsulates the trade-off between their mean and risk. Coherent risk measures have been defined as a particular class of risk measures satisfying certain desirable properties [1]. It has been proved that any coherent risk measure evaluated for some random position can be expressed as the worst-case expectation of this position when the probability measure used in the expectation varies in some uncertainty set, called scenario set [1]. In this way, portfolio optimization using a coherent risk measure is a robust optimization problem. The dual representation of coherent risk measures has been connected to the expected utility theory of von Neumann and Morgenstern [20] through the concept of the Optimized Certainty Equivalent (OCE) [6].

The most well-known coherent risk measure is Conditional Value-at-Risk (CVaR) [21]. CVaR concentrates on the worst-case tail of the position's probability distribution. Portfolio optimization using CVaR is implemented by dualizing its representation via the scenario set [23]. When the probability distribution is unknown but can be restricted to a scenario set containing all the potential distributions, Robust Conditional Value-at-Risk (Robust CVaR) can be defined as the worst-case CVaR when the distribution varies in this set $[11,25]$. The scenario set is constructed by structuring randomness in two stages and concentrating uncertainty at the first stage. Again, dualizing the representation enables portfolio optimization using Robust CVaR $[11,25]$.

Although Robust $\mathrm{CVaR}$ has been shown to be resistent against probability distribution uncertainty, the properties of optimal-Robust CVaR portfolios have not been investigated. Given that portfolio regularization has been proposed to deal with parameter estimation uncertainty, the question of whether optimal-Robust CVaR portfolios possess some kind of regularization property arises.

In this paper, we prove that optimal-Robust CVaR portfolios possess the regularization property. The regularization scheme that they follow is connected with 
the expected utility theory, where the expectation used for the utility is taken with respect to the probability measures that determine the structure of the scenario set. The representation that we prove offers a crisp explanation of the optimal-Robust CVaR portfolio properties with respect to the format and the size of the scenario set.

\section{Robust CVaR}

Consider a set of $m$ financial assets which have random discounted return rates in a one-period horizon. The probability distribution of the returns is uncertain. We describe the stochastic framework. Let $P_{k}, k=1,2, \ldots, r$, and $\mathbb{P}$ be probability measures defined in $\sigma$-field $(\Omega, \mathscr{F})$, such that $P_{k}, k=1,2, \ldots, r$, is absolutely continuous with respect to $\mathbb{P}$. The driving probability measure $P$ is assumed to be a mixture of $P_{k}$ with unknown coefficients. More specifically, the uncertainty in the probability distribution will be determined using a norm $\|\cdot\|$ in $\mathbb{R}^{r}, \xi_{\mathbf{0}}=\left(\xi_{0,1}, \xi_{0,2}\right.$, $\left.\ldots, \xi_{0, r}\right)^{T} \in \mathbb{R}_{+}^{r}$ with $\sum_{k=1}^{r} \xi_{0, k}=1$ and $\phi \in \mathbb{R}_{+}$. If we define the compact set

$$
\Xi:=\left\{\xi=\left(\xi_{1}, \xi_{2}, \ldots, \xi_{r}\right)^{T} \in \mathbb{R}_{+}^{r} \mid\left\|\xi-\xi_{0}\right\| \leq \phi, \sum_{k=1}^{r} \xi_{k}=1\right\},
$$

we have that

$$
P \in S:=\left\{\sum_{k=1}^{r} \xi_{k} P_{k} \mid \xi=\left(\xi_{1}, \xi_{2}, \ldots, \xi_{r}\right)^{T} \in \Xi\right\} .
$$

Set $S$ contains all the potential probability distributions, which reside in an uncertainty set centered at $P_{0}=\sum_{k=1}^{r} \xi_{0, k} P_{k}$. For some level $\beta, 0 \leq \beta<1$, we denote with $\mathrm{CVaR}_{P}(X), \operatorname{RCVaR}(X)$ the $\mathrm{CVaR}$, Robust $\mathrm{CVaR}$, respectively, of some random position $X \in L^{1}(\Omega, \mathscr{F}, \mathbb{P})$ under some probability measure $P \in S$. Random variables $X_{1}, X_{2}, \ldots, X_{m} \in L^{1}(\Omega, \mathscr{F}, \mathbb{P})$ are equal to the discounted return rates of the assets. An investment portfolio over these assets can be represented by $\theta=\left(\theta_{1}\right.$, $\left.\theta_{2}, \ldots, \theta_{m}\right)^{T} \in \mathbb{R}_{+}^{m}$, where $\sum_{j=1}^{m} \theta_{j}=1$. The discounted return rate of the portfolio is $\sum_{j=1}^{m} \theta_{j} X_{j}$.

The portfolio problem using the Robust CVaR criterion is

$$
\begin{aligned}
& \min _{\theta \in \mathbb{R}_{+}^{m}} \operatorname{RCVaR}\left(\sum_{j=1}^{m} \theta_{j} X_{j}\right)=\min _{\theta \in \mathbb{R}_{+}^{m}} \max _{P \in S} \operatorname{CVaR}_{P}\left(\sum_{j=1}^{m} \theta_{j} X_{j}\right) \\
& \text { s.t. } \quad \sum_{j=1}^{m} \theta_{j}=1 \quad \text { s.t. } \quad \sum_{j=1}^{m} \theta_{j}=1 \text {. }
\end{aligned}
$$

We use $\|\cdot\|_{*}$ to denote the dual norm to $\|\cdot\|$. Problem (3) is equivalent [11] to 


$$
\begin{array}{ll}
\min & \eta+t+\phi\|v\|_{*}+\xi_{0}^{T} v \\
\text { s.t. } & t+v_{k} \geq \frac{1}{1-\beta} E_{P_{k}}[Z], k=1,2, \ldots, r \\
& Z \geq-\eta-\sum_{j=1}^{m} \theta_{j} X_{j}, \mathbb{P}-\text { a.s. } \\
& \sum_{j=1}^{m} \theta_{j}=1 \\
& \eta, t \in \mathbb{R}, v \in \mathbb{R}^{r}, Z \in L_{+}^{1}(\Omega, \mathscr{F}, \mathbb{P}), \theta \in \mathbb{R}_{+}^{r} .
\end{array}
$$

Problem (4) can be used to compute optimal-Robust CVaR portfolios. If $\Omega$ is finite, then Problem (4) is a finite-dimensional linear optimization problem and the result follows from linear optimization duality. The derivation of the result is much more difficult in the case that $\Omega$ is infinite. In this case, to solve Problem (4) the Sample Average Approximation or the Stochastic Approximation methods can be used [23]. The resulting portfolio is resistent against varying the probability distribution in the scenario set $S$. Despite that portfolio regularization has been successfully used to protect against parameter estimation uncertainty, it is not known whether optimalRobust CVaR portfolios are regularized.

\section{Regularization of optimal-Robust CVaR portfolios}

The regularization aspect of optimal-Robust CVaR portfolios will be depicted using the expected utility theory of von Neumann and Morgenstern [20] and, in particular, utility function

$$
u(x)=(1-\beta)^{-1} \min (0, x)
$$

where $x \in \mathbb{R}$ is some discounted return rate. Function $u$ imposes a prefential order among financial positions. If $\theta_{\mathbf{1}}=\left(\theta_{1,1}, \theta_{1,2}, \ldots, \theta_{1, m}\right)^{T}, \theta_{\mathbf{2}}=\left(\theta_{2,1}, \theta_{2,2}, \ldots\right.$, $\theta_{2, m}^{T}$ are two asset portfolios, $P \in S$ is the probability measure that drives the asset evolution and $E_{P}\left[u\left(\sum_{j=1}^{m} \theta_{1, j} X_{j}\right)\right] \geq E_{P}\left[u\left(\sum_{j=1}^{m} \theta_{2, j} X_{j}\right)\right]$, portfolio $\theta_{\mathbf{1}}$ is preferred to portfolio $\theta_{2}$.

The next theorem expresses Problem (4) as a trade-off between two functions involving the expected utilities of the investor when present consumption is possible under probability measures $P_{0}, P_{1}, \ldots, P_{r}$. The vector in $\mathbb{R}^{r}$ containing 1 at all positions is denoted by $\mathbf{e}$.

Theorem 1. Problem (4) is equivalent to

$$
\begin{array}{ll}
\min _{\eta, \theta, v} & -E_{P_{0}}\left[\eta+u\left(\sum_{j=1}^{m} \theta_{j} X_{j}-\eta\right)\right]+\phi \min _{t}\|v-t \mathbf{e}\|_{*} \\
\text { s.t. } & v_{k}=-E_{P_{k}}\left[\eta+u\left(\sum_{j=1}^{m} \theta_{j} X_{j}-\eta\right)\right], k=1,2, \ldots, r \\
& \sum_{j=1}^{m} \theta_{j}=1 \\
& \eta \in \mathbb{R}, \theta \in \mathbb{R}_{+}^{m}, v \in \mathbb{R}^{r} .
\end{array}
$$

Proof. Due to lack of space, we present the basic points of the proof. By applying the transformation $\eta:=-\eta, t:=t+\eta$, Problem (4) becomes 


$$
\begin{array}{ll}
\min & \xi_{0}{ }^{T}(v+t \mathbf{e})+\phi\|v\|_{*} \\
\text { s.t. } & v_{k}+t \geq-\eta+\frac{1}{1-\beta} E_{P_{k}}[Z], k=1,2, \ldots, r \\
& Z \geq \eta-\sum_{j=1}^{m} \theta_{j} X_{j}, \mathbb{P}-\text { a.s. } \\
& \sum_{j=1}^{m} \theta_{j}=1, \eta, t \in \mathbb{R}, v \in \mathbb{R}^{r}, Z \in L_{+}^{1}(\Omega, \mathscr{F}, \mathbb{P}), \theta \in \mathbb{R}_{+}^{r} .
\end{array}
$$

Consider an optimal solution $\left(\eta^{o}, t^{o}, v^{o}, Z^{o}, \theta^{o}\right)$ to Problem (7). We claim that it should satisfy

$$
Z^{o}=\max \left(0, \eta^{o}-\sum_{j=1}^{m} \theta_{j}^{o} X_{j}\right) \text { and } v_{k}^{o}+t^{o}=-\eta^{o}+(1-\beta)^{-1} E_{P_{k}}\left[Z^{o}\right] \text { for all } k
$$

Suppose that this is not true. Then, since $\left(\eta^{o}, t^{o}, v^{o}, Z^{o}, \theta^{o}\right)$ is a feasible solution as well, it holds that

$$
Z^{o}>\max \left(0, \eta^{o}-\sum_{j=1}^{m} \theta_{j}^{o} X_{j}\right) \text { or } v_{k}^{o}+t^{o}>-\eta^{o}+(1-\beta)^{-1} E_{P_{k}}\left[Z^{o}\right] \text { for some } k
$$

If we set $Z^{\prime}=\max \left(0, \eta^{o}-\sum_{j=1}^{m} \theta_{j}^{o} X_{j}\right), t^{\prime}=\min _{k}\left\{-v_{k}^{o}-\eta^{o}+\frac{1}{1-\beta} E_{P_{k}}\left[Z^{o}\right]\right\}<$ $t^{o}$, then, $\left(\eta^{o}, t^{\prime}, v^{o}, Z^{\prime}, \theta^{o}\right)$ is feasible with a smaller objective value, which is a contradiction. Thus, the claim is true. Consequently, the inequalities in Problem (7) can be substituted with equalities. The result follows after a few steps.

If the investor decides to consume a fraction $\eta$ of their wealth at present, then, their future return is $\sum_{j=1}^{m} \theta_{j} X_{j}-\eta$ and thus, their total utility is $\eta+u\left(\sum_{j=1}^{m} \theta_{j} X_{j}\right.$ $-\eta$ ) [6]. If $\phi=0$, the problem becomes the portfolio optimization problem using the CVaR criterion, since $E_{P_{0}}\left[\eta+u\left(\sum_{j=1}^{m} \theta_{j} X_{j}-\eta\right)\right]$ is the expected utility with present consumption $\eta$ under probability measure $P_{0}$. If $\phi>0$, the regularization term $\phi \min _{t}\|v-t \mathbf{e}\|_{*}$ is also affecting the portfolio. The $k$-th coordinate $v_{k}$ of the regularization vector $v$ is equal to the opposite of the expected utility with present consumption $\eta$ under probability measure $P_{k}$. Thus, the regularization term is equal to the distance of $v$ from the line $t \mathbf{e}, t \in \mathbb{R}$, according to norm $\|\cdot\|_{*}$. This implies that the optimal-Robust $\mathrm{CVaR}$ portfolios are regularized in such a way that the expected utilities under the probability measures $P_{1}, P_{2}, \ldots, P_{r}$ are close to each other. The metric used to measure their proximity is the dual norm $\|\cdot\|_{*}$ to the norm $\|\cdot\|$ used to define the uncertainty set $S$. This is a different kind of regularization than the ones already considered in the literature $[8,9,13]$. The strength of the regularization is controlled by the size $\phi$ of the uncertainty set $S$.

\section{Conclusions}

We have shown that optimal-Robust CVaR portfolios that deal with a kind of structured uncertainty in the probability distribution that the assets follow possess the regularization property. Since regularized portfolios have already been shown to deal with uncertainty in the parameter estimation, such an observation allows a broader view of the importance of regularization in facing probability distribution uncer- 
tainty. The regularization properties were explicitly connected with the properties of the uncertainty set, enabling the choice of the regularization scheme according to the format and the size of the uncertainty set.

\section{References}

1. Artzner, P., Delbaen, F., Eber, J.-M., Heath, D.: Coherent risk measures. Mathematical Finance 9(3), 203-228 (1999).

2. Black, F., Litterman, R.: Asset allocation: Combining investor views with market equilibrium. Technical Report, Fixed Income Research, Goldman Sachs \& Co., New York (1990).

3. Black, F., Scholes, M.: The Pricing of Options and Corporate Liabilities. Journal of Political Economy 81(3), 637654 (1973).

4. Ben-Tal, A., Nemirovski, A.: Robust convex optimization. Mathematics of Operations Research 23(4), 769-805 (1998).

5. Ben-Tal, A., Nemirovski, A.: Robust solutions of uncertain linear programs. Operations Research Letters 25(1), 1-13 (1999).

6. Ben-Tal, A. Teboulle, M.: An old-new concept of convex risk measures: the optimized certainty equivalent. Mathematical Finance 17(3), 449-476 (2007).

7. Bertsimas, D., Sim, M.: The price of robustness. Operations Research 52(1), 35-53 (2004).

8. Chopra, V. K.: Improving optimization. Journal of Investing 8, 51-59 (1993).

9. DeMiguel, V., Garlappi, L., Nogales, F. J., Uppal, R.: A Generalized Approach to Portfolio Optimization: Improving Performance by Constraining Portfolio Norms. Management Science 55(5), 798-812 (2009).

10. DeMiguel, V., Nogales, F. J.: Portfolio Selection with Robust Estimation. Operations Research 57(3), 560-577 (2009).

11. Fertis, A., Baes, M., Lüthi, H.-J.: Robust Risk Management. Submitted for publication (2011).

12. Frost, P. A., Savarino, J. E.: An empirical Bayes approach to efficient portfolio selection. Journal of Financial and Quantitative Analysis 21, 293-305 (1986).

13. Frost, P. A., Savarino, J. E.: For better performance: Constrain portfolio weights. Journal of Portfolio Management 15, 29-34 (1988).

14. Goldfarb, D., Iyengar, G.: Robust Portfolio Selection Problems. Mathematics of Operations Research 20(1), 1-38 (2003).

15. Huber, P. J.: Robust Statistics. John Wiley \& Sons, New York (1981).

16. Lintner, J.: Valuation of risky assets and the selection of risky investments in stock portfolios and capital budgets. Review of Economics and Statistics 47, 13-37 (1965).

17. Markowitz, H. M.: Portfolio selection. Journal of Finance 7, 77-91 (1952).

18. Merton, R. C.: Theory of Rational Option Pricing. Bell Journal of Economics and Management Science 4(1), 141-183 (1973).

19. Mossin, J.: Equilibrium in capital asset markets. Econometrica 34(4), 768-783 (1966).

20. von Neumann, J., Morgenstern, O.: Theory of Games and Economic Behavior. Princeton University Press, Princeton (1947).

21. Rockafellar, R. T., Uryasev, S.: Optimization of conditional value-at-risk. The Journal of Risk 2(3), 21-41 (2000).

22. Ross, S.: The Arbitrage Theory of Capital Asset Pricing. Journal of Economic Theory 13(3), 341-360 (1976).

23. Shapiro, A., Dentcheva, D., Ruszczynski, A.: Lectures on Stochastic Programming: Modeling and Theory. SIAM, Philadelphia (2009).

24. Sharpe, W.: Capital asset prices: A theory of market equilibrium under conditions of risk. Journal of Finance 19(3), 425-442 (1964).

25. Zhu, S., Fukushima, M.: Worst-case conditional value-at-risk with application to robust portfolio management. Operations Research 57(5), 1155-1168 (2009). 